Research Article

\title{
Prevalence of metallo beta lactamase in clinical isolates of pseudomonas aeruginosa in a tertiary care hospital
}

\author{
Shyamasree Nandy*, Ayan Kumar Das, Mridu Dudeja
}

Department of Microbiology, HIMSR, Jamia Hamdard, New Delhi, India

Received: 19 August 2015

Accepted: 31 August 2015

\section{*Correspondence:}

Dr. Shyamasree Nandy,

E-mail: shyamasree_nandy@yahoo.com

Copyright: ( $)$ the author(s), publisher and licensee Medip Academy. This is an open-access article distributed under the terms of the Creative Commons Attribution Non-Commercial License, which permits unrestricted non-commercial use, distribution, and reproduction in any medium, provided the original work is properly cited.

\section{ABSTRACT}

Background: Increasing Carbapenem resistance in Pseudomonas aeruginosa is an emerging threat and a matter of particular concern. Aim: Our study was conducted to find out the prevalence of Metallo beta lactamase producing Pseudomonas aeruginosa and compare various phenotypic MBL detection methods.

Methods: A prospective study was conducted on 86 non duplicate Pseudomonas aeruginosa strains isolated from clinical specimens for a period of 2 year from April 2011 to march 2013. Total 86 isolates of Pseudomonas aeruginosa were included in the study. All clinical samples were processed according to standard microbiological method. The MIC for Imipenem and Meropenem were determined by broth dilution method. As per CLSI any isolate having an MIC of $>8 \mu \mathrm{g} / \mathrm{ml}$ was considered resistant.42 isolates were both or either resistant to IMP and MRP. These 42 isolates were tested for MBL production by (a) IMP EDTA E test (17 MBL positive isolate detected), (b) IMP \& IMP EDTA disc diffusion test (17 MBL positive isolate detected), (c) IMP \& EDTA double disc synergy test (14 MBL positive isolate detected).

Results: $48.84 \%$ isolates were resistant to Carbapenem and $19.76 \%$ isolates were found to be MBL producer. Colistin showed $100 \%$ susceptibility in all the MBL positive isolates.

Conclusions: Among the 3 test done IMP \& IMP EDTA test is easy to perform, cost effective and as sensitive as E test. Our results strongly suggest that for the MBL isolates should be detected on routine basis and the antibiotic prescribed accordingly.

Keywords: Metallo beta lactamase, Pseudomonas, Carbapenemase

\section{INTRODUCTION}

The first case of plamid mediated MBL in Pseudomonas was reported in 1988 from Japan. ${ }^{1}$ During the early nineties there were only handful cases of MBL production in GNBs. But now reports of carbapenem resistance and MBL production from all over the world has increased manifold. Carbapenems, which were first introduced in 1985, have strong antibiotic activity against beta lactamase producing bacteria. However, they also act as powerful inducers of beta lactamase production. ${ }^{2}$
Pseudomonas aeruginosa in both community and hospital acquired infection is worrisome due to its ability to develop resistance against multiple classes of antibiotics, even during the course of infection. ${ }^{3}$ The main 3 mechanism of antibiotic resistance in Pseudomonas are
a) high inherent resistance
b) Efflux pump
c) enzymatic deactivation of antibiotics.

Moreover it has a unique capability of acquiring resistance rapidly through mutations and transfer of 
genetic elements such as plasmids, transposons and integrons. ${ }^{4}$

Pseudomonas is inherently resistant to many drug classes. It has a gene for Amp C beta lactamase \& hence it is inherently resistant to those beta lactams (e.g., Cephalothin \& Ampicillin) that induce this enzyme and are hydrolyzed by it.

Earlier researchers thought that the impermeability is the reason for antibiotic exclusion. But in early 1990s it was found out that the main reason for antibiotic resistance is Efflux pump. ${ }^{6}$ The efflux pumps are composed of 3 parts that includes an energy dependent pump in cytoplasmic membrane, an outer membrane protein and a linker protein. ${ }^{4}$ Four different antibiotic efflux systems have been described in P.aeruginosa: mexAB-oprM, mexXYoprM, mexCD-oprJ and mexEF-oprN. ${ }^{6}$ All classes of antibiotics except the polymixins are susceptible to exclusion by efflux pump. Pseudomonas also produces various enzymes for inactivation of drugs. For inactivation of Aminoglycosides it produces acetyltransferases (AAC 3I,3II,6'I) \& Adenyltransferases/Nucleotidylntransferases (ANT 2'I). It produces numerous betalactamases among which the 2 most important ones are Carbapenemases \& Extended spectrum beta lactamases (ESBL). ESBL generally confer resistance to all beta lactam drugs except carbapenems (except GES-2). ${ }^{7}$

Carbapenemases are those beta lactamases that significantly hydrolyse at least Imipenem or/and Meropenem. ${ }^{2}$ Based on molecular structure carbapenemases can be divided into 2 groups a) Serine carbapenemases (comprising Group A \& Group D) having a serine moiety at its active site b) Metallobetalactamases (Group B) having zinc ion at its active site. MBL has been further divided into 3 subclasses B1 \& B3 (needs 2 zinc ions for activity) \& B2 (needs 1 zinc ion for its activity). MBL are active against all the beta lactam drugs including cephalosporins \& carbapenems. Generally it cannot hydrolyse monobactam but many IMP \& VIM producing strains have been reported to be resistant towards monobactam. ${ }^{8}$ Till date 6 types of MBL has been described IMP \& VIM (most common and widely spread), SIM, GIM, SPM, AIM (generally localized). Besides these New Delhi Metallo beta lactamase was first reported in 2011 in Pseudomonas from Serbia. ${ }^{9}$ Way back in 1993 there was only one reported case of transferable MBL. Over last 2 decades the cases of MBL production in Pseudomonas has increased significantly. The present study aims to determine the prevalence of MBL producing Pseudomonas in a tertiary care teaching hospital.

\section{METHODS}

A prospective study was conducted on 86 non duplicate Pseudomonas aeruginosa strains which were collected from various clinical specimen including pus, urine, body fluid, blood, sputumetc for a period of 2 years from April 2011 to March 2013. Pseudomonas aeruginosa was identified based on standard laboratory identification protocol that included pigment production, positive Oxidase test, positive citrate utilization test, positive catalase test, positive arginine dihydrolase test and negative Indole, MR, VP test. ${ }^{10}$ Antimicribial susceptibility testing was done in Mueller Hinton Agar by Kirby Bauer disc diffusion method and the result was interpreted as per the 2012 CLSI guidelines. ${ }^{11}$ Antibiotic susceptibility test was done for the following antibiotics Piperacillin, Ceftazidime, Ceftriaxone, Cefperazone, Ceftazidime clavulanic acid, Cefepime, Imipenem, Meropenem, Aztreonam, Chloramphenicol, Levofloxacin, Ciprofloxacin, Gentamycin, Tobramycin, Amikacin, Netillimycin, Colistin.

Preparation of EDTA disc: A 0.5 M EDTA solution was prepared by dissolving $93.05 \mathrm{gm}$. of disodium EDTA . $\mathrm{H}_{2} \mathrm{O}$ (HiMedia Labs), in $500 \mathrm{ml}$ of distilled water. Ph. was adjusted to 8 by adding $\mathrm{NaOH} 12$.The mixture was sterilized by autoclaving. $10 \mu \mathrm{l}$ of $0.5 \mathrm{M}$ EDTA was added to Imipenem disc and Blank disc, dried and stored at 200C for further use. It was seen that EDTA discs retained its efficacy till 16 weeks. EDTA solution can also be stored at -200C.But adding EDTA during every test is a cumbersome process. Hence, we had prepared the discs, stored and had used them within 16 weeks.

The MIC of Imipenem \& Meropenem were determined by broth dilution method. ${ }^{10}$ The isolates that had an MIC $>8 \mu \mathrm{g} / \mathrm{ml}$ were considered as carbapenems resistant. ${ }^{11} 42$ isolates that were either or both resistant to Imipenem or Meropenem were screened for MBL production by following 3 tests.

\section{Imipenem (IMP) -EDTA combined disc test:}

The IMP-EDTA combined disc test was performed as described by Yong et al. ${ }^{12}$ Test organisms (turbidity adjusted to 0.5 McFarlands Standard) were inoculated on to plates with Muller Hinton agar as recommended by the CLSI. $^{11}$ One $10 \mu \mathrm{g}$ Imipenem discs and one Imipenem EDTA was placed. The inhibition zones of the Imipenem and Imipenem EDTA discs were compared 16 to 18 hours of incubation in air at 350C. In the combined disc test, if the increase in inhibition zone with the Imipenem and EDTA disc was $\geq 7 \mathrm{~mm}$ than the Imipenem disc alone, it was considered MBL positive.

\section{Imipenem-EDTA double disc synergy test (DDST):}

The IMP EDTA double disc synergy test was performed as described as described by Lee et al. ${ }^{13}$ Organisms were inoculated (turbidity adjusted to $0.5 \mathrm{M}$ McFarlands Standard) on to plates with Mueller Hinton agar as recommended by the CLSI. ${ }^{11}$ An Imipenem $(10 \mu \mathrm{g})$ disc were placed $20 \mathrm{~mm}$ center to center from another EDTA disc $(750 \mu \mathrm{g})$. Enhancement of the zone of inhibition in the area between Imipenem and the EDTA disc in 
comparison with the zone of inhibition on the far side of the drug was interpreted as a positive result.

\section{MBL E Test:}

The Eteststrip (Himedia Laboratories) with IMP (4 to 256 $\mu \mathrm{g} / \mathrm{ml}$ ) and IMP EDTA (1 to $64 \mu \mathrm{g} / \mathrm{ml})$ were applied on Muller Hinton agar and were incubated for 16 to $20 \mathrm{hrs}$. at 350C.The presence of MBL was indicated by a reduction of $\mathrm{MIC}$ by $\geq 3$ doubling dilutions in the presence of EDTA.

\section{RESULTS}

Table 1: Nature and source of isolates.

\begin{tabular}{|llll|}
\hline $\begin{array}{l}\text { Source of } \\
\text { Isolates }\end{array}$ & $\begin{array}{l}\text { Total } \\
\text { number of } \\
\text { isolates }\end{array}$ & $\begin{array}{l}\text { Carbapenem } \\
\text { Resistant } \\
\text { isolates }\end{array}$ & $\begin{array}{l}\text { MBL } \\
\text { positive }\end{array}$ \\
\hline ICU & 39 & 20 & 07 \\
\hline Medicine & 16 & 07 & 02 \\
\hline Surgery & 19 & 09 & 05 \\
\hline Gynae & 08 & 04 & 02 \\
\hline Paediatrics & 04 & 02 & 01 \\
\hline Total & 86 & $42(48.84 \%)$ & $17(19.76 \%)$ \\
\hline
\end{tabular}

Table 2: Comparison of 3 phenotypic tests.

\begin{tabular}{|lll|}
\hline $\begin{array}{l}\text { Imipenem EDTA } \\
\text { double disc } \\
\text { synergy test }\end{array}$ & $\begin{array}{l}\text { Imipenem EDTA } \\
\text { combined disc } \\
\text { test }\end{array}$ & MBL E test \\
\hline 14 & 17 & 17 \\
Sensitivity:82.35\% & Sensitivity:100\% & Sensitivity:100\% \\
\hline
\end{tabular}

Table 3: Antibiotic Susceptibility pattern of MBL producing \& non-producing Carbapenem resistant isolates of Pseudomonas aeruginosa.

\begin{tabular}{|lll|}
\hline Antibiotics & $\begin{array}{l}\text { MBL } \\
\text { positive(n=17) }\end{array}$ & $\begin{array}{l}\text { MBL } \\
\text { negative(n=25) }\end{array}$ \\
\hline Piperacillin & $0(0 \%)$ & $3(12 \%)$ \\
\hline Ceftazidime & $0(0 \%)$ & $4(16 \%)$ \\
\hline Ceftriaxone & $0(0 \%)$ & $4(16 \%)$ \\
\hline Cefperazone & $0(0 \%)$ & $4(16 \%)$ \\
\hline $\begin{array}{l}\text { Ceftazidime } \\
\text { clavulanic acid }\end{array}$ & $0(0 \%)$ & $5(20 \%)$ \\
\hline Cefepime & $0(0 \%)$ & $4(16 \%)$ \\
\hline Imipenem & $0(0 \%)$ & $0(0 \%)$ \\
\hline Meropenem & $2(11.76 \%)$ & $3(12 \%)$ \\
\hline Aztreonam & $4(23.53 \%)$ & $3(12 \%)$ \\
\hline Chloramphenicol & $1(05.8 \%)$ & $1(4 \%)$ \\
\hline Levofloxacin & $4(23.53 \%)$ & $3(12 \%)$ \\
\hline Ciprofloxacin & $1(05.8 \%)$ & $2(8 \%)$ \\
\hline Gentamycin & $3(17.65 \%)$ & $7(28 \%)$ \\
\hline Tobramycin & $2(11.76 \%)$ & $2(08 \%)$ \\
\hline Amikacin & $4(23.53 \%)$ & $8(32 \%)$ \\
\hline Netillimycin & $2(11.76 \%)$ & $2(08 \%)$ \\
\hline Colistin & $17(100 \%)$ & $25(100 \%)$ \\
\hline
\end{tabular}

\section{DISCUSSION}

A total of 86 isolates were collected for a period of 2 years. Among these 42 isolates were found to be resistant towards both Imipenem and Meropenem or resistant towards imipenem and sensitive towards meropenem. 5 of them were resistant to Imipenem but sensitive to Meropenem. So we had included 42 Imipenem resistant isolates and 5 Meropenem sensitive Imipenem resistant isolates. Among Meropenem sensitive isolates 2 were found to be MBL producing.

Carbapenem resistance with increasing frequency is been reported from various parts of India. The percentage of MBL production among the carbapenem resistant isolates was found to be varying. Some studies found it to be as high as $97 \% .{ }^{14}$ We have found that $48.8 \%$ isolates were resistant to carbapenems among which $40.47 \%$ were MBL producer. This indicates that other mechanism (such as efflux pump) is more frequent mechanism for resistance towards carbapenems. Other studies conducted in Delhi by Behera et $\mathrm{al}^{15}$, Singh et $\mathrm{al}^{16}$ and Sahaet $\mathrm{al}^{17}$ shows the percentage of MBL production in Pseudomonas aeruginosa to be respectively $76.19 \%$, $94.7 \%$, \& $61 \%$ among Carbapenem resistant isolates. In our case the percentage of MBL production in Carbapenem resistant isolates were much lower.

Out of these 42 isolates 14 were found to be MBL producer by all 3 methods, 17 were positive for MBL by IMP EDTA disc potentiation test and Etest. The efficacy of Imipenem EDTA disc potentiation test was found to be equally comparable to that of MBL Etest. The sensitivity of Imipenem EDTA double disc synergy test was found to be quite lower $(82.24 \%)$ than the other 2 tests. Though chromosomal MBL is yet not reported in Pseudomonas sp., varieties of transferable MBL is being reported with increasing frequency. The most widely distributed IMP \& VIM genes are mostly located in Integron I (some IMP genes are found in class III integrin also). ${ }^{18}$ The most worrisome factor is generally IMP \& VIM genes are located in integrons carrying several other resistance genes (eg: Resistant genes for Aminoglycosides). ${ }^{7}$ As a result these isolates show co resistance phenotypes. In our study we have found that MBL positive isolates are showing a very high resistance to various groups of drugs other than beta lactams. Out of 4 aminoglycosides (Amikacin, Gentamycin, Netillimycin \& Tobramycin) the best result was observed with Amikacin (23.53\%).Sensitivity towards Chloramphenicol was almost negligible (5.8\%). Among the quinolones Levofloxacin showed better susceptibility (23.53\%) compared to Ciprofloxacin (5.8\%). This indicates that most of the MBL producing isolates included in our study were carrying multi resistance genes.

\section{CONCLUSION}

Our study was intended to find out the prevalence of MBL in Pseudomonas aeruginosa which we found to be 
$19.76 \%$ of total isolates. The patient we cater to mostly belong to weaker socioeconomic background who has limited access to healthcare. May be this is the reason for the lower rate of carbapenem resistance compared to the other studies conducted in Delhi.

Till date CLSI has not given any guidelines on which test to follow for diagnosis of MBL. We had performed 3 tests and among them we found IMP \& IMP EDTA double disc combined test to be equally sensitive as IMP EDTA E test. As E test is very costly it is not economically feasible to conduct this regularly. Instead IMP \& IMP EDTA double disc test can use which is cheap and easy to perform. Our study and all other studies also indicate that generally MBL producing isolates carry multidrug resistant integrons. Infact the only drug that showed $100 \%$ susceptibility towards MBL producing isolates was Colistin.Hence, identification and AST of MBL producing Pseudomonas aeruginosa is essential for proper prophylaxis.

\section{Funding: No funding sources}

Conflict of interest: None declared

Ethical approval: The study was approved by the Institutional Ethics Committee

\section{REFERENCES}

1. Watanabe $\mathrm{M}$, Iyobe $\mathrm{S}$, Inoue $\mathrm{M}$, Mitsuhashi S. Transferable imipenem resistance in Pseudomonas aeruginosa. Antimicrob Agents Chemother. 1991;35:147-51.

2. Nordmann P, L. Poirel."Emerging carbapenemases in Gram-negative aerobes. "Clinical Microbiology and Infection 2002;8(6):321-31.

3. Lister, Philip D, Wolter DJ, Hanson ND. Antibacterial-resistant Pseudomonas aeruginosa: clinical impact and complex regulation of chromosomally encoded resistance mechanisms." Clinical Microbiology Reviews. 2009;22(4):582610.

4. Livermore DM. Multiple mechanisms of antimicrobial resistance in Pseudomonas aeruginosa: our worst nightmare? Clinical Infectious Diseases. 2002;34(5):634-40.

5. Livermore DM. Beta-Lactamases in laboratory and clinical resistance." Clinical microbiology reviews 1995;8(4):557-84.

6. Poole K. "Multidrug efflux pumps and antimicrobial resistance in Pseudomonas aeruginosa and related organisms." Journal of molecular microbiology and biotechnology 2001;3(2):255-64.

7. Mesaros N, Nordmann P, Plesiat RD, Van Eldere J, Glupczynski Y, Laethem YV, et al. "Pseudomonas aeruginosa: resistance and therapeutic options at the turn of the new millennium." Clinical microbiology and infection 2007;13(6):560-78.
8. Walsh, Timothy R., et al. "Metallo- $\beta$-lactamases: the quiet before the storm?" Clinical microbiology reviews 18.2 (2005):306-325.

9. Jovcic B., Lepsanovic Z., Suljagic V., Rackov G., Begovic J., Topisirovic L., \& Kojic M.. Emergence of NDM-1 metallo- $\beta$-lactamase in Pseudomonas aeruginosa clinical isolates from Serbia. Antimicrobial agents and chemotherapy, 55(8), (2011),3929-3931.

10. Bailey W. E. \&Scott E. G. (1962). Diagnostic microbiology. Diagnostic microbiology.

11. Clinical and Laboratory Standards Institute (CLSI), Performance standards for antimicrobial susceptibility testing, 22nd informational supplement (M100-S22), Vol 32, No.3, Jan 2012.

12. Yong D., Lee K., Yum J. H., Shin H. B., Rossellini G. M., \& Chong Y. (2002). Imipenem-EDTA disk method for differentiation of metallo- $\beta$-lactamaseproducing clinical isolates of Pseudomonas spp. and Acinetobacter spp. Journal of Clinical Microbiology, 40(10), 3798-3801.

13. Lee K., Lim Y. S., Yong D., Yum J. H., \& Chong Y. (2003). Evaluation of the Hodge test and the imipenem-EDTA double-disk synergy test for differentiating metallo- $\beta$-lactamase-producing isolates of Pseudomonas spp. and Acinetobacter spp. Journal of Clinical Microbiology,41(10),46234629.

14. Gupta V., Singla N., \& Chander J. Use of two double disk synergy tests to detect metallo beta lactamases in nonfermenters. Indian Journal of Medical Research, 128(5), 2008).671.

15. Behera B., Mathur P., Das A., Kapil A., \& Sharma V. (2008). An evaluation of four different phenotypic techniques for detection of metallo- $\beta$ lactamase producing Pseudomonas aeruginosa. Indian Journal of medical microbiology, 26(3),233.

16. Singh SP, Shariff M, Barua T, Thukral SS. Comparative evaluation of phenotypic tests for identification of metallo beta-lactamases producing clinical isolates of Pseudomonas aeruginosa. Indian J Med Res. 2009;129:713-5.

17. Saha R, Jain S, Kaur IR. Metallo beta-lactamase producing pseudomonas species--a major cause of concern among hospital associated urinary tract infection. Journal of the Indian Medical Association, 2010;108(6):344-8.

18. Walsh TR, Toleman MA, Poirel L, Nordmann P. Metallo- $\beta$-lactamases: the quiet before the storm? Clinical microbiology reviews. 2005;18(2):306-25.

Cite this article as: Nandy S, Das AK, Dudeja M. Prevalence of metallo beta lactamase in clinical isolates of pseudomonas aeruginosa in a tertiary care hospital. Int J Community Med Public Health 2015;2:566-9. 